\title{
Electrophysiological outcomes after spinal cord injury
}

\author{
James Xie, ${ }^{1,2}$ And MaXwell Boakye, M.D. ${ }^{1}$ \\ ${ }^{1}$ Neural Plasticity and Outcomes Research Laboratories, Veterans'Affairs Palo Alto Health Care System, and \\ Department of Neurosurgery, Stanford University School of Medicine; and ${ }^{2}$ Stanford University, Stanford, \\ California
}

\begin{abstract}
Electrophysiological measures can provide information that complements clinical assessments such as the American Spinal Injury Association sensory and motor scores in the evaluation of outcomes after spinal cord injury (SCI). The authors review and summarize the literature regarding tests that are most relevant to the study of SCI recovery - in particular, motor evoked potentials and somatosensory evoked potentials (SSEPs). In addition, they discuss the role of other tests, including F-wave nerve conductance tests and electromyography, sympathetic skin response, and the Hoffman reflex (H-reflex) test as well as the promise of dermatomal SSEPs and the electrical perceptual threshold test, newer quantitative tests of sensory function.

It has been shown that motor evoked potential amplitudes improve with SCI recovery but latencies do not. Somatosensory evoked potentials are predictive of ambulatory capacity and hand function. Hoffman reflexes are present during spinal shock despite the loss of tendon reflexes, but their amplitudes increase with time after injury. Further, H-reflex modulation is reflective of changes in spinal excitability. While these tests have produced data that is congruent with clinical evaluations, they have yet to surpass clinical evaluations in predicting outcomes. Continuing research using these methodologies should yield a better understanding of the mechanisms behind SCI recovery and thus provide potentially greater predictive and evaluative power. (DOI: 10.3171/FOC.2008.25.11.E11)
\end{abstract}

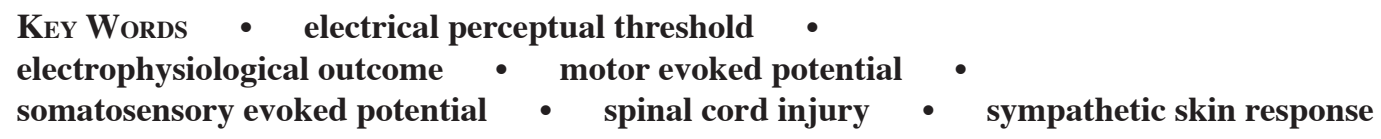

$\mathrm{O}$ VER the past decade, electrophysiological assessments of functional outcomes after SCI have become more popular and better documented. These assessments include MEP recordings, SSEP recordings, dSSEP recordings, EMG recordings, SSR recordings, Hreflex recordings, and EPT recordings. With these tools, researchers hope to be able to accurately determine level and density of SCI, provide reliable monitoring of recovery over spinal cord segments, and provide indices of function..$^{12}$

Electrophysiological measures are able to offer a number of advantages over qualitative clinical measures. Firstly, electrophysiological recordings provide quantitative, objective data that can be analyzed by blinded researchers. ${ }^{31}$ Secondly, the measures are more flexible and environment independent, thus allowing researchers to perform recordings on unresponsive, uncooperative,

\footnotetext{
Abbreviations used in this paper: ASIA = American Spinal Injury Association; cSCI = complete SCI; dSSEP = dermatomal SSEP; $\mathrm{EMG}=$ electromyography; $\mathrm{EPT}=$ electrical perceptual threshold; H-reflex = Hoffmann reflex; iSCI = incomplete SCI; MEP = motor evoked potential; SCI = spinal cord injury; SCIM = Spinal Cord Independence Measure; SSEP = somatosensory evoked potential; SSR $=$ sympathetic skin response; tSSEP = tibial SSEP; WISCI = Walking Index for Spinal Cord Injury.
}

or comatose patients. ${ }^{15}$ Thirdly, measures of evoked potentials complement existing SCI recovery assessments, such as the ASIA sensory and motor scores, as they are able to assess specific parts of the spinal segments and peripheral nerve tracts. In particular, measures can target specific spinal segments below the level of injury. ${ }^{13} \mathrm{Fi}-$ nally, combinations of recording techniques can provide detailed quantitative information about a patient's condition that cannot be determined through other clinical means. ${ }^{4}$ There is much promise in using these measures to assess SCI, predict functional outcomes, and inform clinicians about the planning and results of therapeutic interventions.

Despite the benefits of these measures, however, they have yet to be fully standardized and validated in clinic, and there is a need for further research and detailed guidelines. ${ }^{31}$ The relationship between changes in electrophysiological measures and different quantifiers of recovery has not been fully explored yet, and the mechanisms of recovery from iSCI and cSCI are also still in need of further investigation. Much remains unknown about how these electrophysiological measures correlate to this recovery.

\section{Motor Evoked Potentials}

Motor evoked potentials provide a means for assess- 


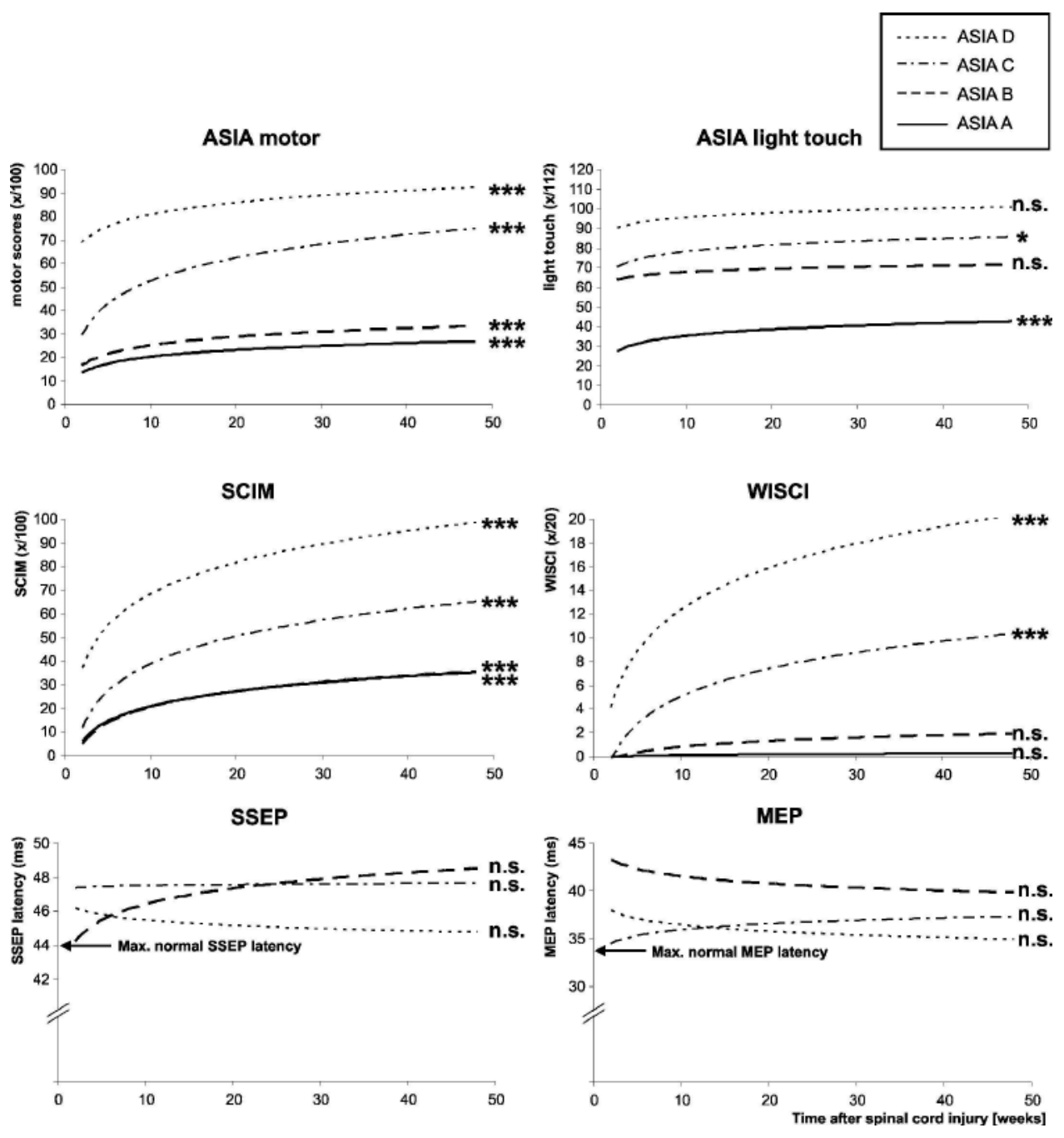

FIG. 1. Course of neurological, functional, and neurophysiological measures after SCI. One-year follow-up of neurological sensory-motor scores (ASIA motor and light touch), functional outcome measures (SCIM and WISCI), and neurophysiological assessments of SSEP and MEP latencies in tetraplegic subjects with motor-sensory complete (ASIA Score A, bold line), sensory incomplete but motor complete (ASIA Score B, striped bold line), and motor-sensory incomplete (ASIA Score C, striped and dotted line, and ASIA Score D, dotted line) SCI. Asterisks indicate the significance of change from baseline to 48 weeks after SCI $\left({ }^{\star} p<0.05,{ }^{* \star} p<0.01,{ }^{* *} p<0.001\right)$. n.s. = not significant. Note the different scales of the y axis for SSEP and MEP plots. In patients with abolished SSEP and MEP responses, latencies could not be calculated. Maximal reference SSEP and MEP latency values (healthy volunteers) were derived from Buchner H, Noth J: Evoked Potentials, Neurovegetative Diagnostic, Oculographia: Methods and Clinical Application. Georg Thieme Verlag: Stuttgart, 2005. Figure and legend (with adaptation) reprinted with permission. The publisher for this copyrighted material is Mary Ann Liebert, Inc., Publishers.

ment of descending spinal tract function. They are induced by transcranial magnetic stimulation of the motor cortex and recorded on muscles of interest using surface electrodes to determine the level and extent of the SCI lesion. ${ }^{4}$ Recordings of MEP latencies are thought to reflect the speed of conduction down the corticospinal tract; changes may suggest the level of remyelination/regeneration/reconnection of the corticospinal tract over time, and MEP amplitudes have been found to correlate with muscle movement velocity. ${ }^{32}$

Wirth et al. ${ }^{32}$ recently published results of a 6-month study of patients with iSCI, investigating changes in corti- cospinal function through recording of torque-controlled MEPs and ankle motor control. They found that the percentage increase in MEP amplitude was significantly related to the percentage increase of maximal movement velocity of the ankle. However, MEP latencies remained delayed during the 6-month period, a result that is consistent with findings of past studies. ${ }^{6,29}$ Also of note, MEP amplitudes were found to have a high intraclass correlation coefficient $(0.81)$ within healthy subjects, thus confirming MEP amplitude reliability. The use of MEPs as an accurate diagnostic tool is consistent with previous research findings. ${ }^{10}$ 


\section{Electrophysiological outcomes after spinal cord injury}

In a large multicenter study, Curt et al. ${ }^{7}$ published findings tracking the functional recovery mechanisms of compensation, neural plasticity, and repair of the central nervous system over a 1-year period. As illustrated in Fig. 1, they again found that evoked potential latencies remained unchanged over time, indicating that functional recovery in SCI patients was not related to improvements in spinal conductivity. Overall, improvements in function as assessed by the WISCI and SCIM scores did not correlate with reductions in evoked potential latencies. Therefore it was concluded that the findings strongly support the idea that recovery mainly occurs through compensation in cSCI and through facilitation by neural plasticity in iSCI rather than through physical repair.

It has also been found that task-dependent modulation of MEPs is a valuable extension to the use of MEPs as a diagnostic monitoring tool in iSCI. Diehl et al. ${ }^{11}$ assessed tibialis anterior muscle response to MEPs induced by transcranial magnetic stimulation in patients with iSCI, finding a similar task-dependent modulation to that found in upper limb MEPs. ${ }^{1}$ Studying dynamic versus static motor tasks adds an additional degree of diagnostic depth in monitoring motor function.

\section{Somatosensory Evoked Potentials}

Somatosensory evoked potentials provide a means for assessment of ascending spinal tract function. They are generated by stimulating peripheral nerves and recording the response from the patient's scalp. As a prognostic tool, SSEPs have been found to have predictive value in determining ambulation outcomes, although not to a degree more accurate than conventional clinical examination. ${ }^{18}$

Additional studies have validated the use of SSEPs as an assessment tool. Curt et al..$^{5}$ showed a positive relationship between median and ulnar nerve SSEP amplitude and outcome of hand function as well as an overall capability for SSEPs to assess level of injury. Curt et al. ${ }^{3}$ further showed a correlation between ASIA motor scores obtained in patients with SCI and their SSEP measures as a predictor of future ambulation, thus further establishing the value of SSEP as a diagnostic tool. Somatosensory evoked potentials have also been used to assess differences between patients with ischemic SCI and those with traumatic SCI. Iseli et al. ${ }^{17}$ found that both groups of patients had similar motor and sensory deficits and both groups exhibited pathological SSEP recordings. Tibial SSEP recordings in patients with ischemic SCI and both pudendal SSEP and tSSEP recordings in patients with traumatic SCI had predictive power in assessing patient recovery. In a recent study, Spiess et al. ${ }^{30}$ also confirmed the use of tSSEPs as a predictor of functional and neurological outcomes. This study also highlighted the possible fragility of electrophysiological recordings, citing spontaneous changes to recordability, latencies, and amplitudes in tSSEPs, and recommended taking this variability into account when using tSSEPs in studies.

\section{Dermatomal SSEPS}

Dermatomal SSEPs are SSEPs elicited with cutane- ous electrical stimulation of ASIA sensory points. Shields et al. ${ }^{28}$ were able to record dSSEPs in all dermatomes of 10 volunteers but were only able to elicit them in 5 of 9 patients with SCI and concluded that dSSEPs were of limited value in determining the level of cervical SCI. In a recent study of 34 patients with cervical SCI, Kramer et al. ${ }^{20}$ showed a good correlation between pathological EPT values and both abolished and pathological dSSEPs.

\section{Electromyography and Nerve Conductance Studies}

Electromyography is often used with other tests to record elicited responses. In conjunction with nerve conductance studies, such as the F-wave measures, EMG is good for assessing whether there has been an injury to peripheral nerves. F-waves are elicited following supramaximal stimulation of a peripheral nerve and can be used to assess the efferent peripheral motor pathway. Fwaves have also been used to assess the excitability of the spinal motor neuron pool. ${ }^{16,22,23}$

\section{Sympathetic Skin Response}

The SSR is another noninvasive electrophysiological assessment that records the response in the skin surface by EMG electrodes from electric or magnetic stimulation above the spinal lesion. ${ }^{4}$ Physiological (auditory and inspiratory gasp) stimulation can also be used to elicit SSR. ${ }^{24}$ These recordings can be used to assess damage to the spinal sympathetic nervous system and the respective peripheral sympathetic nerve fibers connecting the recorded skin areas. Some of the main pathways tested are palmar (testing the pathway from spinal cord to the sweat glands of the hands), plantar (pathway from spinal cord to feet), and perineal (from spinal cord to pre- and postganglionic sympathetic nerve fibers). ${ }^{8}$

Cariga et al. ${ }^{2}$ investigated SSR generation in an isolated spinal cord and the relationship between SSR, levels of SCI lesion, and supraspinal connections. They found that SSR could not be generated by an isolated spinal cord. Furthermore, in patients with cSCI, SSR was completely absent at any neurological level below the lesion, while in those with iSCI, SSR was dependent on the preservation of supraspinal connections. Thus, these connections are necessary for SSR, specifically along with central sympathetic pathways of upper thoracic segments for palmar SSR, and possibly all thoracic segments for plantar SSR. The authors also concluded that while SSR has not traditionally been used to provide an assessment for completeness of SCI lesion as much as somatic tests such as MEPs and SSEPs have been, the addition of sympathetic nervous system tests may provide a more complete picture for studying SCI.

Building upon this idea, Nicotra et al. ${ }^{24}$ set out to study whether SSR provides a good measure of spinal sympathetic pathways in SCI. Indeed, SSR did provide a method for assessing integrity of sympathetic cholinergic pathways in patients with SCI, and it also provided responses that related with the ASIA impairment scale. Accordingly, SSR may provide another key component for assessing SCI that the current ASIA impairment scale lacks and could be another assessment to consider when 
monitoring and evaluating recovery and interventions. Nevertheless, SSR tests for this purpose have yet to be investigated thoroughly.

\section{Hoffmann Reflex}

The H-reflex test is the electrical analog of the monosynaptic stretch reflex. It can be elicited by low intensity (submaximal) electrical stimulation of the afferent fibers of a mixed peripheral nerve such as the tibial nerve or the common peroneal nerve. Afferent nerve stimulation leads to activation of the $\alpha$-motor neuron, which is recorded using a surface EMG electrode. The H-reflex is generally considered to be a measure of motor neuron excitability. Changes in H-reflex amplitudes as a result of an intervention or a conditioning stimulus can be used to probe spinal cord excitability and pathophysiology. ${ }^{19}$ The H-reflex amplitudes are increased after SCI. ${ }^{26}$ During spinal shock, H-reflexes are present despite the loss of tendon reflexes. Over time, H-reflex amplitudes increase after SCI. ${ }^{14}$ Modulation of the H-reflex is a reflection of ongoing changes in spinal cord physiology and plasticity. Thus far there have not been any studies in which lasting behavioral changes have been induced from H-reflex modulation, although that is an area of intense research. ${ }^{25}$

\section{Electrical Perceptual Thresholds}

The EPT test is a noninvasive quantitative sensory test developed to assess the level and degree of impairment of SCI. The test mimics light touch or pinprick tests with cutaneous electrical stimulation of ASIA sensory points along spinal dermatomes. ${ }^{9}$ Savic et al. ${ }^{27}$ validated the use of this test as a simple and reproducible means of testing in patients with SCI. Level of SCI as determined by EPT was found to be as accurate as clinical assessment according to ASIA classifications. Moreover, the authors concluded that EPT may add sensitivity and resolution to clinical testing that could be used in monitoring patients with SCI in clinical investigations.

\section{Conclusions}

Electrophysiological measures are increasingly being used to provide objective measures for SCI assessment. They are able to provide predictive value with a degree of significance similar to that provided by clinical examinations using ASIA scoring and provide informative, quantitative data on the changes that occur in neural circuitry. ${ }^{21}$ Used in conjunction with conventional clinical examinations, electrophysiological examinations have come to be a good complement for assessing function after SCI. Furthermore, the tests themselves also complement each other in providing a broader picture of the condition. Ultimately, determining the underlying significance of these measures may provide a better understanding of the way in which neural plasticity, repair, compensation, and improvement interrelate in SCI.

\section{Disclaimer}

The authors report no conflict of interest concerning the materials or methods used in this study or the findings specified in this paper.

\section{References}

1. Aranyi Z, Mathis J, Hess CW, et al: Task-dependent facilitation of motor evoked potentials during dynamic and steady muscle contractions. Muscle Nerve 21:1309-1316, 1998

2. Cariga P, Catley M, Mathias CJ, et al: Organisation of the sympathetic skin response in spinal cord injury. J Neurol Neurosurg Psychiatry 72:356-360, 2002

3. Curt A, Dietz V: Ambulatory capacity in spinal cord injury: significance of somatosensory evoked potentials and ASIA protocol in predicting outcome. Arch Phys Med Rehabil 78:39-43, 1997

4. Curt A, Dietz V: Electrophysiological recordings in patients with spinal cord injury: significance for predicting outcome. Spinal Cord 37:157-165, 1999

5. Curt A, Dietz V: Traumatic cervical spinal cord injury: relation between somatosensory evoked potentials, neurological deficit, and hand function. Arch Phys Med Rehabil 77:4853, 1996

6. Curt A, Keck ME, Dietz V: Functional outcome following spinal cord injury: significance of motor-evoked potentials and ASIA scores. Arch Phys Med Rehabil 79:81-86, 1998

7. Curt A, Van Hedel HJ, Klaus D, et al: Recovery from a spinal cord injury: significance of compensation, neural plasticity, and repair. J Neurotrauma 25:677-685, 2008

8. Curt A, Weinhardt C, Dietz V: Significance of sympathetic skin response in the assessment of autonomic failure in patients with spinal cord injury. J Auton Nerv Syst 61:175-180, 1996

9. Davey NJ, Nowicky AV, Zaman R: Somatopy of perceptual threshold to cutaneous electrical stimulation in man. Exp Physiol 86:127-130, 2001

10. Di Lazzaro V, Oliviero A, Profice P, et al: The diagnostic value of motor evoked potentials. Clin Neurophysiol 110:12971307, 1999

11. Diehl P, Kliesch U, Dietz V, et al: Impaired facilitation of motor evoked potentials in incomplete spinal cord injury. J Neurol 253:51-57, 2006

12. Ellaway PH, Anand P, Bergstrom EM, et al: Towards improved clinical and physiological assessments of recovery in spinal cord injury: a clinical initiative. Spinal Cord 42:325337, 2004

13. Fawcett JW, Curt A, Steeves JD, et al: Guidelines for the conduct of clinical trials for spinal cord injury as developed by the ICCP panel: spontaneous recovery after spinal cord injury and statistical power needed for therapeutic clinical trials. Spinal Cord 45:190-205, 2007

14. Hiersemenzel LP, Curt A, Dietz V: From spinal shock to spasticity: neuronal adaptations to a spinal cord injury. Neurology 54:1574-1582, 2000

15. Houlden DA, Schwartz ML, Klettke KA: Neurophysiologic diagnosis in uncooperative trauma patients: confounding factors. J Trauma 33:244-251, 1992

16. Inghilleri M, Lorenzano C, Conte A, et al: Effects of transcranial magnetic stimulation on the $\mathrm{H}$ reflex and $\mathrm{F}$ wave in the hand muscles. Clin Neurophysiol 114:1096-1101, 2003

17. Iseli E, Cavigelli A, Dietz V, et al: Prognosis and recovery in ischaemic and traumatic spinal cord injury: clinical and electrophysiological evaluation. J Neurol Neurosurg Psychiatry 67:567-571, 1999

18. Jacobs SR, Yeaney NK, Herbison GJ, et al: Future ambulation prognosis as predicted by somatosensory evoked potentials in motor complete and incomplete quadriplegia. Arch Phys Med Rehabil 76:635-641, 1995

19. Knikou M: The H-reflex as a probe: pathways and pitfalls. J Neurosci Methods 171:1-12, 2008 


\section{Electrophysiological outcomes after spinal cord injury}

20. Kramer JL, Moss AJ, Taylor P, et al: Assessment of posterior spinal cord function with electrical perception threshold in spinal cord injury. J Neurotrauma 25:1019-1026, 2008

21. Lim PA, Tow AM: Recovery and regeneration after spinal cord injury: a review and summary of recent literature. Ann Acad Med Singapore 36:49-57, 2007

22. Lin JZ, Floeter MK: Do F-wave measurements detect changes in motor neuron excitability? Muscle Nerve 30:289-294, 2004

23. Lukacs M: F wave measurements detecting changes in motor neuron excitability after ischaemic stroke. Electromyogr Clin Neurophysiol 47:109-115, 2007

24. Nicotra A, Catley M, Ellaway PH, et al: The ability of physiological stimuli to generate the sympathetic skin response in human chronic spinal cord injury. Restor Neurol Neurosci 23:331-339, 2005

25. Perez MA, Lungholt BK, Nielsen JB: Short-term adaptations in spinal cord circuits evoked by repetitive transcranial magnetic stimulation: possible underlying mechanisms. Exp Brain Res 162:202-212, 2005

26. Petersen NT, Pyndt HS, Nielsen JB: Investigating human motor control by transcranial magnetic stimulation. Exp Brain Res 152:1-16, 2003

27. Savic G, Bergstrom EM, Frankel HL, et al: Perceptual threshold to cutaneous electrical stimulation in patients with spinal cord injury. Spinal Cord 44:560-566, 2006

28. Shields CB, Ping Zhang Y, Shields LB, et al: Objective as- sessment of cervical spinal cord injury levels by transcranial magnetic motor-evoked potentials. Surg Neurol 66:475-483, 2006

29. Smith HC, Savic G, Frankel HL, et al: Corticospinal function studied over time following incomplete spinal cord injury. Spinal Cord 38:292-300, 2000

30. Spiess M, Schubert M, Kliesch U, et al: Evolution of tibial SSEP after traumatic spinal cord injury: baseline for clinical trials. Clin Neurophysiol 119:1051-1061, 2008

31. Steeves JD, Lammertse D, Curt A, et al: Guidelines for the conduct of clinical trials for spinal cord injury (SCI) as developed by the ICCP panel: clinical trial outcome measures. Spinal Cord 45:206-221, 2007

32. Wirth B, Van Hedel HJ, Curt A: Changes in corticospinal function and ankle motor control during recovery from incomplete spinal cord injury. J Neurotrauma 25:467-478, 2008

Manuscript submitted July 14, 2008.

Accepted August 14, 2008.

Address correspondence to: Maxwell Boakye, M.D., Neuroplasticity Laboratory, Department of Neurosurgery, Stanford University Medical Center/Palo Alto VA, 3801 Miranda Avenue, M-112, Palo Alto, California, 94304. email: mboakye@stanford. edu. 